\title{
Error simulations of uncorrected NDVI and DCVI during remote sensing measurements from UAS
}

\begin{abstract}
Remote sensing from unmanned aerial systems (UAS) has been gaining popularity in the last few years. In the field of vegetation mapping, digital cameras converted to calculate vegetation index (DCVI) are one of the most popular sensors. This paper presents simulations using a radiative transfer model (libRadtran) of DCVI and NDVI results in an environment of possible UAS flight scenarios. The analysis of the results is focused on the comparison of atmosphere influence on both indices. The results revealed uncertainties in uncorrected DCVI measurements up to $25 \%$ at the altitude of $5 \mathrm{~km}, 5 \%$ at $1 \mathrm{~km}$ and around $1 \%$ at $0.15 \mathrm{~km}$, which suggests that DCVI can be widely used on small UAS operating below $0.2 \mathrm{~km}$.
\end{abstract}

Keywords

Remote sensing $\cdot$ vegetation index $\cdot$ digital camera $\cdot$ UAS $\cdot$ atmospheric correction

(C) University of Warsaw - Faculty of Geography and Regional Studies

\author{
Michał T. Chiliński \\ Marek Ostrowski ${ }^{2}$ \\ 'Department of Geoinformation and Remote Sensing \\ Faculty of Geography and Regional Studies \\ University of Warsaw, \\ Institute of Geophysics \\ University of Warsaw \\ e-mail: mich@igf.fuw.edu.pl \\ 2Laboratory of Image-based Information and Modelling \\ Faculty of Biology \\ University of Warsaw \\ e-mail: samper@samper.pl \\ Received: 14 November 2013 \\ Accepted: 8 April 2014
}

Introduction

The last decade has been a period of rapid increase in the application of the lightweight unmanned aerial system (UAS) in areas such as science, farming and emergency management (Hunt et al. 2005; Lelong et al. 2008; Nebiker et al. 2008; Rango et al. 2009; Hardin \& Hardin 2010; Xiang \& Tian 2011; Zahng \& Kovacs 2012). Today, UAS are cheap, very mobile, and easy to pilot, enabling extremely quick preparation of measurements and low operational costs. From this perspective, it is the best method for measurements with a lightweight sensor for low altitudes. UAS are a solution for aerial imagery in restricted areas of developed countries, where full-size research planes are prohibited; they also make it possible to conduct measurements from the sky in developing countries, which do not have sufficient resources for a fleet of full-size planes.

Due to the smaller size of UAS in comparison to regular research planes, they can carry a smaller payload, which means that many aerial sensors cannot be used in UAS. The costs of development and production of remote sensing devices are significant, making end-user prices higher than the costs of flying platforms. In such a situation, a common practice is using simpler sensors, i.e. home-made ones, made from mass production units.

An example of a sensor made from a standard consumer product is a digital camera, without an IR filter (hot mirror), equipped with a colour filter, with the ability to register the near infrared spectrum range, which can be used for the calculation of a vegetation condition index. The vegetation index calculated from the data obtained from a digital camera is a differential normalized index, calculated in the same way as the Normalized Differential Vegetation Index (NDVI) (Rouse et al. 1973); however, instead of the infrared and red channels, the infrared and blue channels are used. Digital cameras usually have a colour filter array (Bayer filter) which splits the CCD/CMOS matrix into a mosaic of blue, green and red pixels, which usually works in the following ranges: $400-525,500-625,600-700$ [nm] with overlapping regions. After the removal of the IR-filter, red pixels register 600-825 [nm] both red and IR channels. To receive two separated channels, colour filters which block light between $\sim 450-700 \mathrm{~nm}$ are used (for example: SCHOTT BG3, Rosco \#2007 VS BLUE). Pictures taken with such filters have the IR channel registered on red pixels, the blue channel on blue pixels and the green pixels are dark (as a separation between channels). During a conference where methods of calculating the vegetation index from digital cameras were presented, many scientists (XX Polish Conference on Photointerpretation and Remote Sensing, Warsaw, 2010; XXI Polish Conference on Photointerpretation and Remote Sensing, Toruń, 2012) disagreed on applying the name of NDVI to both indices. Therefore, for the purpose of this paper the vegetation index calculated from digital camera pictures with the infrared and blue channel is called the Digital Camera Vegetation Index (DCVI). In Figure 1 examples of DCVI pictures are presented.

The modifications to digital cameras required for acquiring DCVI mosaics are easy and inexpensive. Moreover, they can usually be made by scientists themselves, although certain companies offer ready-modified devices. The availability of these cameras is increasing and their popularity is growing constantly. The increase in DCVI usage, however, does not entail the development of special software for processing the pictures and the RAW data for the purpose of calculating the 


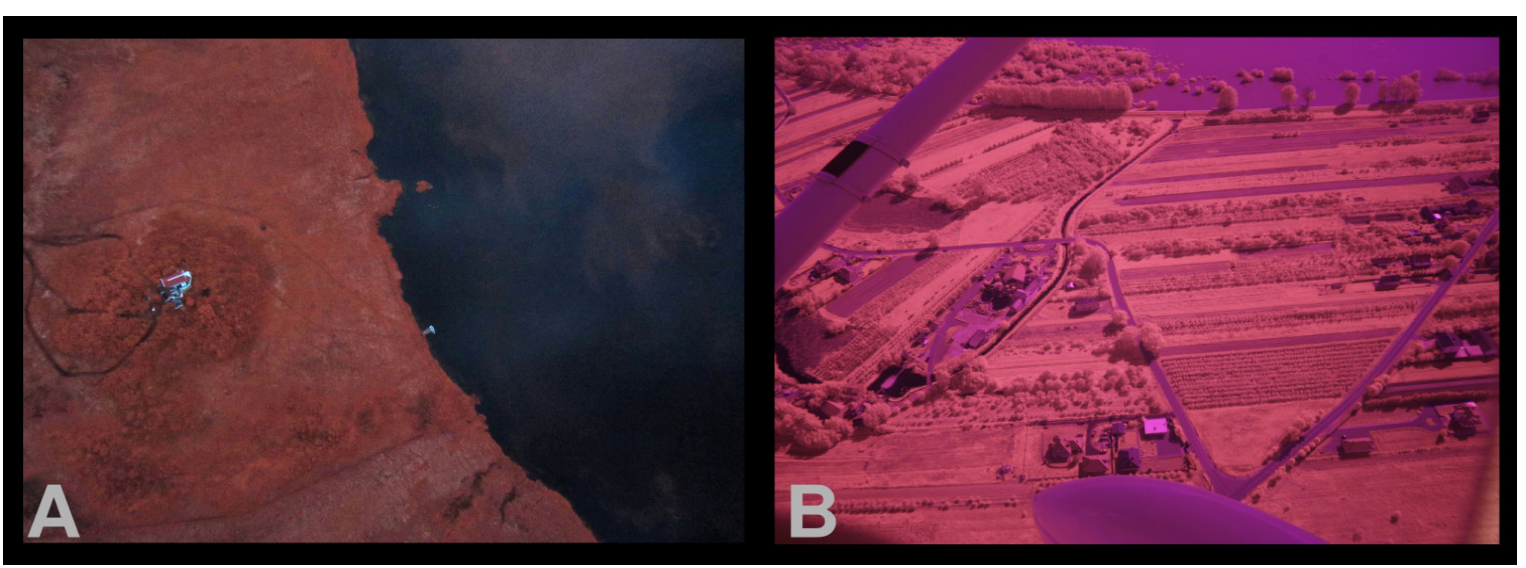

Figure 1. Examples of DCVI pictures taken from UAS (A: tundra) and full-sized plane (B: agricultural area). Photos by M. Chiliński

vegetation indices. Hence, their users have to prepare their own programming solutions, such as script examples from the camera modifiers, or simple codes extracted from the Internet (Fisher et al. 2012). The pictures from UAS are primarily used for orthophotomosaics, and thus there is software on the market dedicated to mosaicking and orthorectification of images from non-photogrametric cameras. However, there is still a significant problem with the use of remote sensing software packages for performing radiometric corrections and removing the influence of the atmosphere on measurements. This leads to a situation in which most DCVI mosaics are calculated without any atmospheric corrections.

This paper describes the results of simulations with a radiative transfer model, presents the possible impact of the atmosphere on DCVI mosaics, shows how varied that influence is for different altitudes, and focuses on the geometry of sun-target-sensor. The main goal is to compare the sensitivity of DCVI and NDVI for the interaction of radiation with the atmosphere, and to determine the parameter areas where the use of DCVI without any corrections can give reliable results.

NDVI is one of the simplest and most popular remote sensing methods used for the assessment of vegetation condition. It is based on the difference between the red and infrared radiances reflected by the vegetation. The amount of radiation in both channels depends on the biophysical characteristics of the vegetation. To ensure the data was independent of the sun zenith angle, the calculated index was normalized by dividing it by the sum of radiance in both of the used channels (Formula 1).

$N D V I=\frac{\lambda_{N I R}-\lambda_{R E D}}{\lambda_{N I R}+\lambda_{R E D}}$

NDVI has values from -1 to 1 , where values around -1 correspond to water, values around 0 correspond to bare soil, urban areas and rocks, and positive values above 0.3 represent vegetation. Lower values $(0.3-0.5)$ are common for grasslands and lower vegetation. Higher values (up to 1 ) represent intensive green areas, such as temperate and tropical rain forests. The biological foundations of NDVI are based on the very high reflectance of vegetation in the near infrared $(750-950 \mathrm{~nm})$, and strong absorption in the red $(650-700 \mathrm{~nm})$. The reflectance in the infrared for vegetation is five times greater than in the visible spectra, where the highest reflectance is observed in the green. When a plant becomes dry, its cell structure changes, which leads to a decrease of reflectance in the near infrared. On the other hand, when chlorophyll does not receive important nutrients, it starts to degrade, which is visible in the reduced absorption in the red spectra and in the increase in reflectance. Since it's invention NDVI has been very intensively used for monitoring the condition and cover of vegetation from many types of satellite and airborne sensors (Tucker et al. 1986; Running 1990; Myneni et al. 1997; Ichii et al. 2001; Burgheimer et al. 2006). Figure 2a presents the reflectance of a fresh standardized leaf and how it changes at 4 , 7 and 24 hours after picking. In the same figure, the MSS bands used to calculate NDVI are presented. DCVI uses exactly the same formula, but with the blue channel instead of the red one, which is depicted in Figure $2 \mathrm{~b}$.

There are three main types of interaction between radiation and matter: absorption, emission and scattering (diffraction, refraction, reflection), all of which lead to a change in propagation direction. One effect of absorption is the weakening intensity of radiation passing through matter. In this process, energy can be absorbed only in strictly defined quanta, depending on the frequency of the radiation (Formula 2).

$E=h v=h \frac{c}{\lambda}$

Different molecules, owing to their composition, absorb different energy photons, which are usually represented by the characteristic absorption spectra distinct for every substance. Different characteristic regions are found in UV, visible, infrared, TIR or microwaves. The composition of the Earth's atmosphere results in the presented absorption spectra (Figure 3). It can be noted that the clear atmosphere is nearly $100 \%$ transparent in the 400-700 nm range, known as visible light.

The process of scattering leads to a change in radiation propagation direction. There are two types of scattering: (1) elastic, where the frequency of scattered radiation is the same as before the scattering; and (2) nonelastic, during which a change of frequency occurs. In the case of remote sensing, elastic scattering is dominant. The characteristics of scattering depend on a unit-less parameter, i.e. the ratio of the scattering particle size and the wavelength of the resulting radiation (Formula 3 ).

$x=\frac{2 \pi r}{\lambda}$

Rayleigh scattering occurs in a situation where $x<<1$. The insensitivity of the scattered radiation in the Rayleigh process is inversely proportional to the fourth power of the wavelength, which leads to a substantially greater scattering for shorter wavelengths (mainly the UV and blue light) (Formula 4). The radiation of the sky (excluding the sun's surface) is only scattered; as a result, due to Rayleigh scattering, the colour of the sky is blue. Rayleigh scattering is anisotropic (a slight deviation from isotropic) and is symmetrical in the forward/backward direction. 
a)

b)

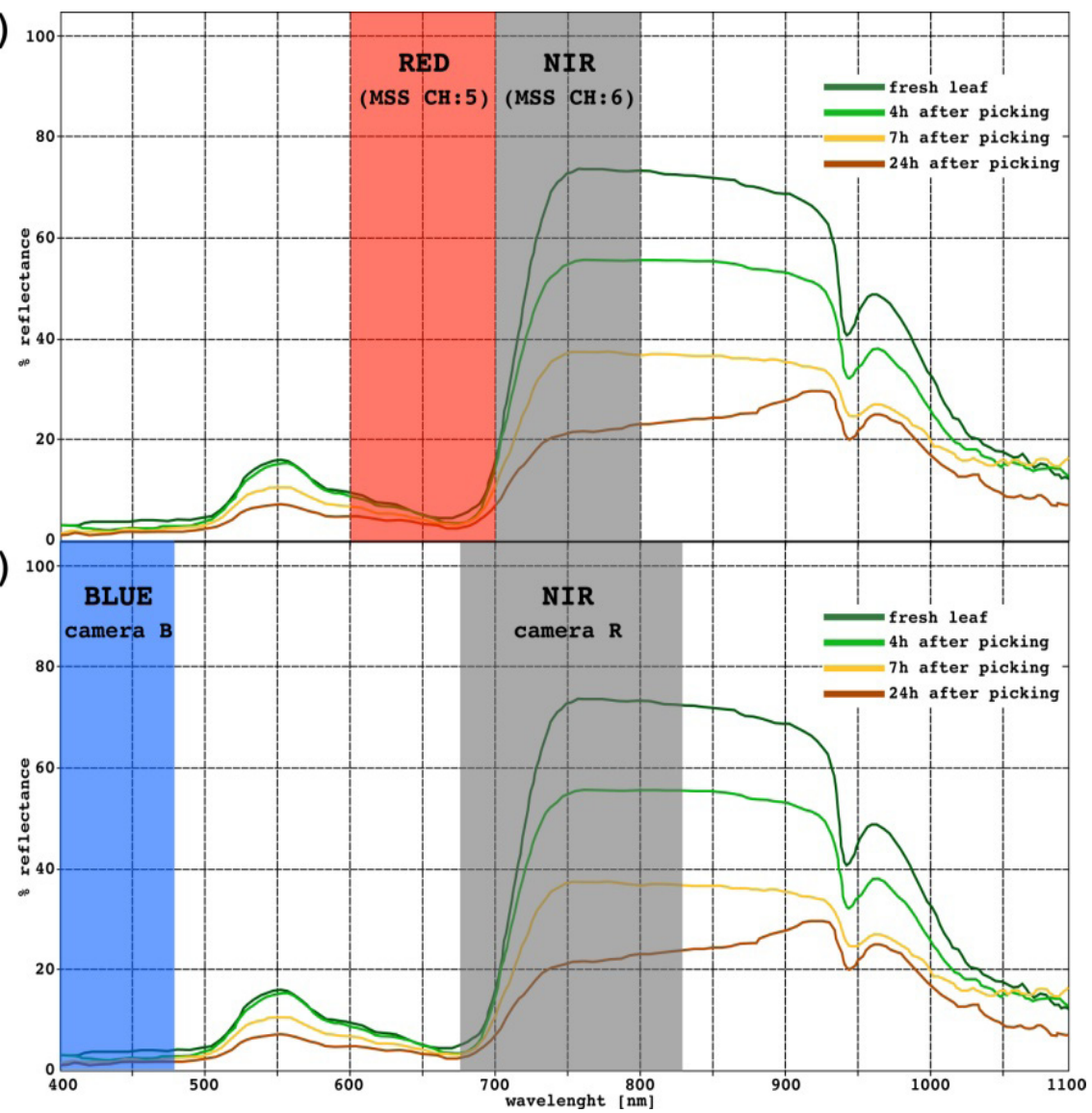

Figure 2. Standardized leaf reflectance with spectral bands of MSS sensor (NASA 2013) (a) and DCVI digital camera (b). Note. The data are from (Chiliński 2010)

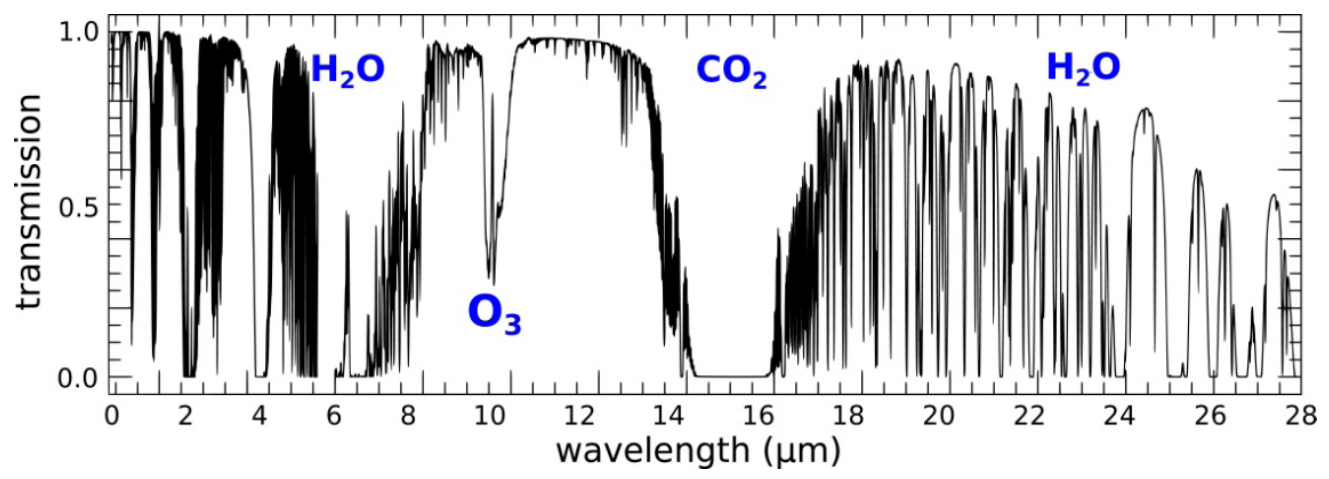

Figure 3. Atmosphere transmission and absorption of different gases. Note. The data are from (HITRAN 2012)

$$
I=I_{0} \frac{1+\cos ^{2} \theta}{2 R^{2}}\left(\frac{2 \pi}{\lambda}\right)^{4}\left(\frac{n^{2}-1}{n^{2}+2}\right)^{2}\left(\frac{d}{2}\right)^{6}
$$

Mie scattering is characteristic for $x>1$ particles, which have a size similar to scattered radiation. This type of scattering is characteristic for visible light and clouds. Mie scattering usually remains constant for different wavelengths and this is what makes the colour of clouds white, as their shade is a mixture of light of different wavelengths.

Geometrical scattering is used to describe the scattering of particles $x>>1$, which are larger than the wavelength of the incidental radiation. In such a situation, the process of scattering is based on the general geometric optics rules.
The behaviour of radiation on particles is described by a set of parameters. The phase function describes the probability of scattering for any solid angle. Larger particles scatter more in a forward direction, whereas scattering from smaller particles is more homogeneous. The phase function is given by (Formula 5 ).

$p(\cos \Theta) \equiv \frac{\sigma(\cos \Theta)}{\frac{1}{4 \pi} \int_{4 \pi} \sigma(\cos \Theta) d \omega},\left[s r^{-1}\right]$

The Rayleigh phase function is symmetrical in the forward and backward direction, and has less radiation scattered in the area perpendicular to the beam.

The scattering coefficient describes how much radiation is removed from a flux on a single path measured by the scattering- 
unit $[1 / \mathrm{m}]$. The absorption coefficient is similar, but in that case radiation is removed by absorption. The sum combination of those two coefficients provides us with the extinction coefficient. The single scattering albedo (SSA) is the ratio between the scattering and extinction coefficients, and it oscillates around the range $0-1$, where 0.9 means that 9 photons from every 10 are scattered and only 1 is absorbed. Optical depth is a parameter describing the change in the intensity of radiation passing through the medium, and is proportional to the physical thickness and optical properties of that medium (Formula 6).

$\Delta \tau=\sigma_{e} \Delta z$

Aerosols also have a significant influence on the propagation of radiation in the atmosphere, thus special models were developed, which characterize the physical and optical properties of the particles in a given air-column. Every model differs in composition: its elements come from different categories, such as mineral dust, sea salt, organic matter (pollens), soot or chemical compounds. Sometimes the optical properties of aerosols depend on temperature and humidity, mainly when there are a lot of hygroscopic compounds. Not only does composition influence the transmission of radiation, but an important role is also played by vertical profiles, which affect the distribution of the scattered and absorbed radiation at different layers of the atmosphere. The main types of aerosols with different mixtures and size distributions are as follows: maritime, urban, rural and desert. To physically describe the behaviour of radiation in the atmosphere with aerosols, a radiative transfer equation with a multiple scattering and absorption module should be applied (Formula 7).

$\mu \frac{d I_{v}}{d \tau}=-I_{v}+(1-\omega) B_{v}+\frac{\omega}{4 \pi} \int I\left(\theta^{\prime}, \varphi^{\prime}\right) P\left(\theta, \varphi ; \theta^{\prime}, \varphi^{\prime}\right) \sin \theta^{\prime} d \theta^{\prime} d \varphi^{\prime}$

The complexity of this integro-differential equation leads to the extensive usage of numerical radiative transfer models in modelling the influences of aerosols on radiative transfer. For remote sensing products, all available information about aerosols is crucial to enable the removal of their impact from the Earth observation remote sensing data. Simulations with radiative transfer models are a very effective method for investigating potential impact on remote sensing data and potential corrections of such data.

\section{Methods}

The entire data for this analysis came from simulations carried out with libRadtran (libRatran 2013), a library of radiative routines and programs. The most important part of the package is the radiative transfer tool called uvspec, designed to calculate irradiance and actinic flux in the full solar and thermal spectrum, from $120 \mathrm{~nm}$ to $100 \mu \mathrm{m}$ (Kylling 1992). Uvspec is specially designed to provide a user-friendly file-driven configuration interface, where a variety of options may be set up: the atmosphere type together with molecules, aerosols, clouds with different water and ice content, or the properties of the boundary layer. As a numerical solver for the radiative transfer equation, the cdisort (a type of DISORT) was used (Stamnes et al. 1988). It was translated to the C-programming language by Timothy E. Dowling. The radiation field in the Earth's atmosphere was calculated with the uvspec tool, which derives its parameters (temperature, humidity and aerosol profiles) from predefined databases, or user-defined input. Boundary conditions were given by the solar spectrum at TOA and the surface at ground level, which could be defined by albedo or BRDF (from a database or user-defined). The simulation with uvspec was divided into three parts. To begin with, atmospheric parameters such as pressure, gas profiles and cloud parameters were converted into optical properties and thus became input for the second stage, where the radiative transfer equation solver calculated the irradiance, actinic fluxes and heating rates for the given parameters. The last stage involved post-processing of the solver output, involving EarthSun distance correction and integration over wavelengths. The results of those computations were presented in the output file, which consisted of the following parameters: wavelength, direct beam irradiance, diffuse-down irradiance, diffuse-up irradiance, direct beam contribution to mean intensity, diffuse-downward contribution to mean intensity, and diffuse-upward contribution to mean intensity. The key questions of the research were answered based on the results calculated with the help of the model with varying inputs for geometry, wavelength, altitude, aerosol type the aerosol optical depth.

For every run of the model, certain parameters were appropriately adjusted. The ozone content in the air column was set at the universal value of 300DU. The sensor zenith angle was set at 0 degrees (nadir), a value common in aerial mapping. The chosen atmosphere type definition was characteristic for mid-latitude summertime. The model already had six predefined standard atmospheres types, as defined by Anderson et al. (1986). The type definitions include profiles of the following eight properties: pressure, temperature, air density, ozone density, oxygen density, water vapour density, $\mathrm{CO}_{2}$ density and $\mathrm{NO}_{2}$ density. An overview of the parameters used in the model is presented in (Table 1).

Additional explanation is required for the albedo and aerosol type parameters. Albedo, defined as grass, introduced to the model the wavelength dependency of a standardized lambertian surface. The surface was lambertian, however the albedo at different wavelengths imitated the albedo of common vegetation. The changes in grass albedo with wavelengths are depicted in (Figure 4)

The Aerosol Type parameter describes the vertical profile (load) and composition of aerosols in the atmosphere. To determine the influence of aerosols on measurements, two aerosol mixtures were chosen. The aerosol type was determined with the Optical Properties of Aerosols and Clouds (OPAC) database (Hess et al. 1998). Every aerosol type in the OPAC database is constructed as a mixture of ten different aerosol compounds, which are: insoluble, water soluble, soot (black carbon), sea salt accumulation mode, sea salt coarse mode, mineral nucleation mode, mineral accumulation mode, mineral coarse mode and mineral transported and sulphate droplets. For the following analysis, it was decided to use urban and continental average aerosol types, which are most likely for the region of Poland. The composition of both aerosol types is depicted in (Figure 5).

The preparation of input files and processing of output from libRadtran was performed in the Python programming language, with the NumPy package for multi-dimensional scientific computing. The graphs and visualizations were prepared with matplotlib, a python 2D plotting library.

\section{Results}

For the simulations, the initial phase was always selected and analysed separately, since during this phase various scenarios were tested in order to find which of the variables contribute significantly to the results of vegetation indices. These scenarios were calculated separately for all variables, including the fixed ones. To aid understanding, the figures present merged plots for different runs, where modifications to the fixed variables were carried out. Each simulation was performed for both of the analysed indices - NDVI and DCVI. Figure 6 presents the results where the influence of different altitudes in the range $0-5 \mathrm{~km}$ for $\mathrm{NDVI} / \mathrm{DCVI}$ was examined. The simulation was conducted using 
Table 1. List of the significant parameters, both constant and varying, during different model runs

\begin{tabular}{|c|c|c|}
\hline Input Parameter & Range & Units \\
\hline Wavelength & 469 (blue), 650 (red), 800 (nir) & Julian Days \\
\hline Day of year & $0-365$ & DU \\
\hline Ozone Column & 300 & ppm \\
\hline CO $_{2}$ & 350 & unitless \\
\hline Albedo & Grass & degrees \\
\hline Solar Zenith Angle & $0-60$ & degrees \\
\hline Solar Azimuth Angle & 0 & degrees \\
\hline View Zenith Angle & 0 (nadir) & km \\
\hline Altitude & $0-5$ & unitless \\
\hline AOD & $0-0.25$ & -- \\
\hline Aerosol Type & Urban, rural & -- \\
\hline Atmospheric type & Midlatitude Summer/Winter & \\
\hline
\end{tabular}

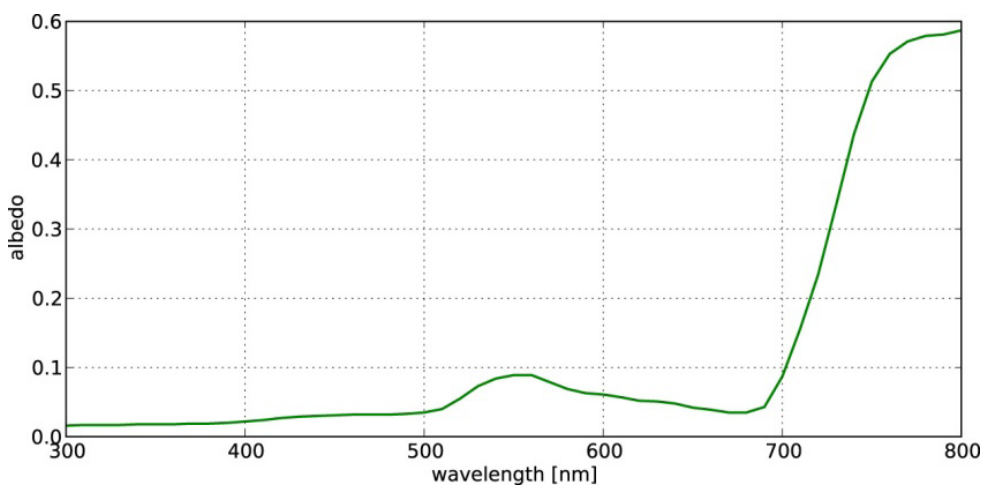

Figure 4. Grass spectral albedo used during simulations.

Note. The data are from (Wanner et al. 1997)

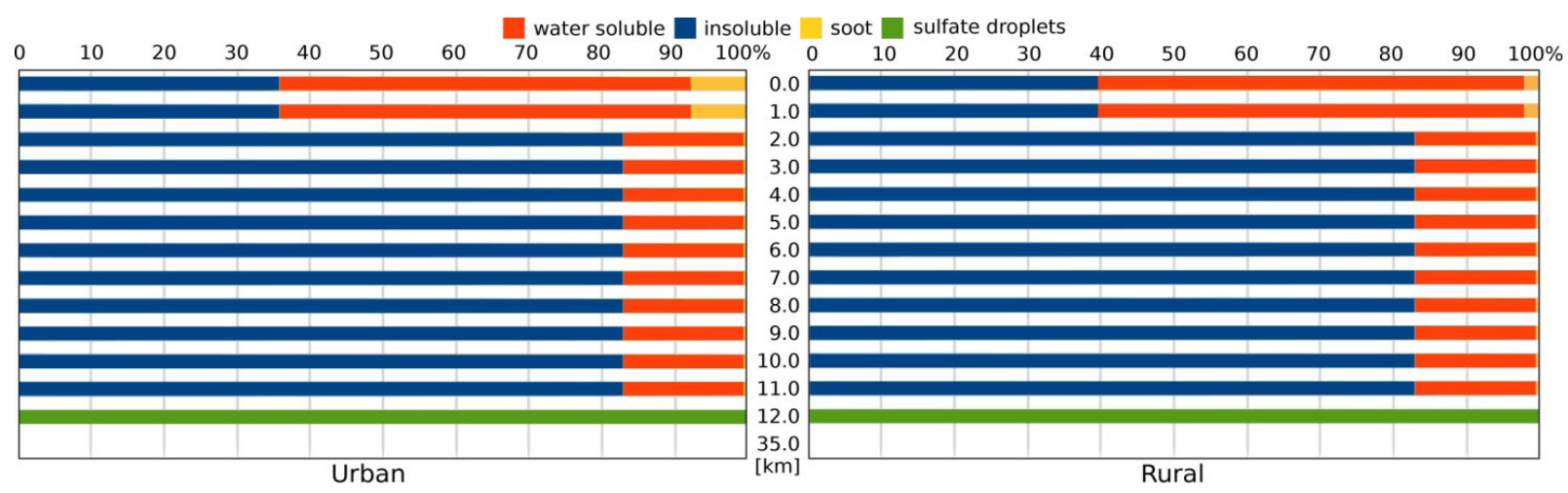

Figure 5. Composition of urban and rural aerosol mixture based on the OPAC database.

Note. The data are from (Hess et al. 1998)

the solar zenith angle (SZA) fixed to 0 degrees and three different aerosol optical depths (AOD). The type of aerosol mixture was fixed as the urban type. The presented figure confirms the high correlation between NDVI and DCVI, which is practically independent from AOD, with only a slight deviation around ground level (altitude $0 \mathrm{~km}$ ). As altitude increases, we observe the constant decrease of the index values. The decrease in DCVI is three times greater than for NDVI. NDVI decreased from 0.83 to 0.77 for $A O D=0.2$, while $D C V I$ dropped from the same stat value to 0.64 . The linear increase of $A O D$ also increased the fall ratio of the vegetation indices. In the first two kilometres of the atmosphere, where there is the highest load of aerosols of the whole vertical profile, the decrease of $\mathrm{DCVI} / \mathrm{NDVI}$ is steepest. For the red lines presenting $A O D=0$, the decrease ratio is constant over the entire profile. This set of simulations confirmed the high influence of altitude on the results. 


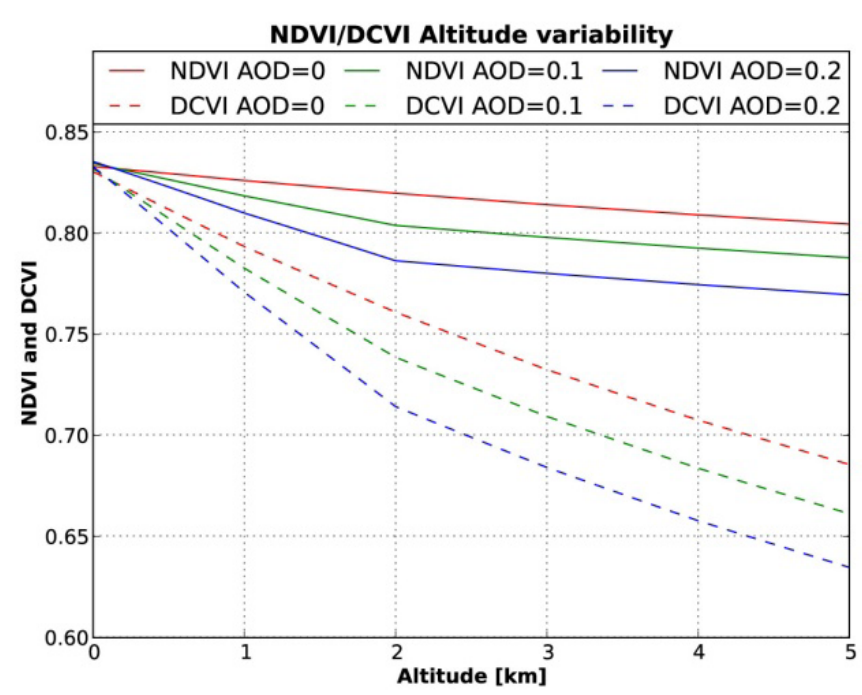

Figure 6. NDVI/DCVI variability with altitude

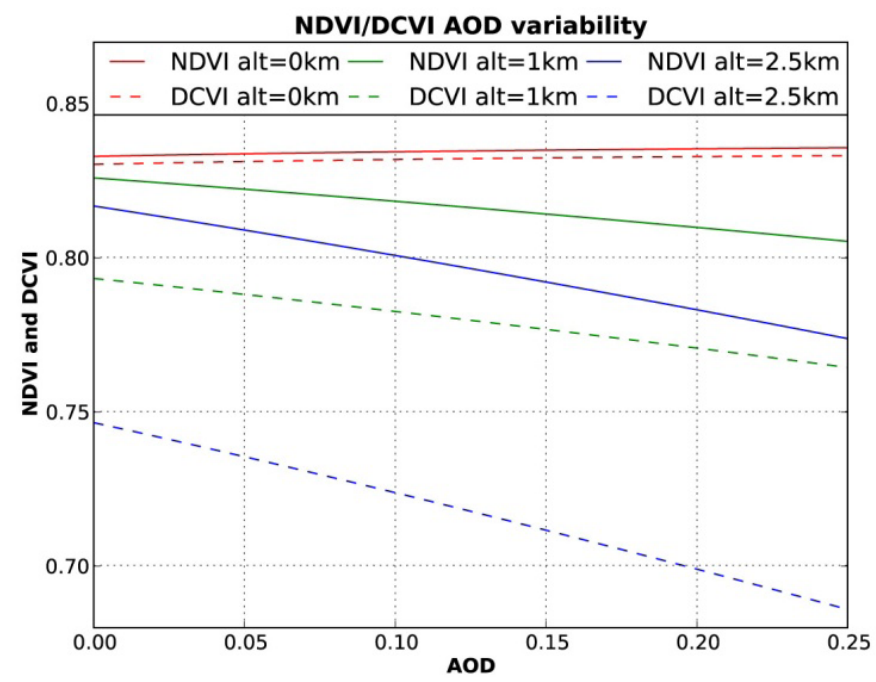

Figure 7. NDVI/DCVI variability with $A O D$

The next set of simulations was conducted to evaluate the entire range of common AODs, as observed during average conditions (Zawadzka et al. 2013) (Figure 7). Parameters for the simulations were exactly the same as in the previous case, but the analysed variable was an AOD with three predefined altitudes. As expected, at ground level we observed constant values of $\mathrm{NDVI} / \mathrm{DCVI}$, independent from the amount of aerosols in the atmosphere. The differences between them are less than $1 \%$. At higher altitudes, we observe a decrease in the index values with the simultaneous increase of AOD. The decrease ratio is slightly quicker with a higher $A O D$ at higher altitudes; nevertheless, in the range AOD $0-0.25$, it is nearly linear with only a $0.38 \%$ deviation from the linearity. As in the first analysed set, DCVI is much more sensitive to changes in AOD and altitude. The discrepancy in NDVI between $A O D=0$ at ground level and $A O D=0.25$ at 2.5 is only $7 \%$; for $\mathrm{DCVI}$ it is more than double for the same points, at $18 \%$.
The assumption made for the vegetation surface used for the calculations was that we had an ideal diffusely reflecting surface with spectral characteristics similar to theoretical grass. Lambertian surfaces should reflect the same quantity of radiation in every direction, regardless of the direction of incoming radiation flux. To test this assumption, a third simulation set was programmed, where SZA varied from 0 degrees (zenith) to 60 degrees, which covers most of the possible SZAs during the day/ year when aerial imaging is performed (Figure 8). As for the AOD set, the model was run for three different altitudes. We expected a higher sensitivity of DCVI for altitude changes, similar to the earlier runs. NDVI values up to $S Z A=40$ degrees were almost constant, which confirmed our theory. Above, we see a slight increase in NDVI value at ground level and at $1 \mathrm{~km}$ altitude. The results for $2.5 \mathrm{~km}$ present a hardly visible decrease in values above $50 \mathrm{deg}$. The structure of the DCVI results was similar, but with a higher decrease/increase of values, which became visible above $30 \mathrm{deg}$. The increase of index values at ground 


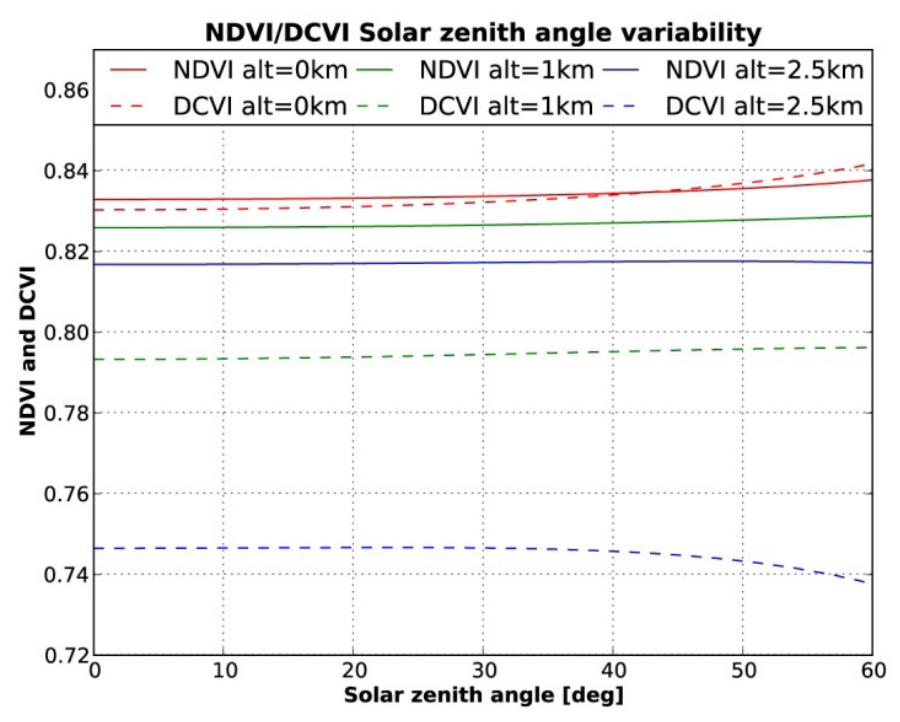

Figure 8. NDVI/DCVI variability with Solar Zenith Angle

level is due to the longer optical path for radiation when the Sun is setting. This longer optical path means that there is a higher probability of Rayleigh scattering for shorter wavelengths (the same mechanism is responsible for red sunsets). NDVI/DCVI had higher values when the difference between the two analysed bands was greater. The radiation from the red and blue bands was more scattered than from the near infrared, and this led to an increase in the vegetation indices. The blue band, as compared to the red band, was more sensitive to Rayleigh scattering, and thus DCVI saw a greater increase in values at ground level than NDVI. On the other hand, at higher altitudes, where the mass of the atmosphere between the Sun and the target is smaller, the NDVI was mostly constant. In the case of DCVI, at higher altitudes we observed the effect of scattering on the path between the target and the sensor, which resulted in a slight decrease in values for SZA angles above 40 degrees.

At the end of that stage of the simulations, a comparison of two aerosol types was performed. Of the two selected aerosol compositions, one was characteristic for an urban area, and the other for a rural one. This simulation was conducted to determine which aerosol type had a greater influence on the results and what the relative differences between them were, in the analysed range of $A O D$, altitudes and SZA. On that basis, we made a decision about whether it was necessary to perform further analysis for both aerosol types separately or if the difference was insignificant enough to make calculations for only one. Figure 9 presents that in the range of altitudes up to $2.5 \mathrm{~km}$ and AODs up to 0.25 , the highest difference for DCVI was $2.04 \%$, while for NDVI it was only $0.65 \%$. For both, the vegetation index differences increased up to $A O D=0.05$ linearly, and after that point they showed a weakly visible exponential dependence with AOD. Urban aerosols have a higher amount of black carbon and its highly absorbing particles. The absorption was the same for the entire wavelength spectrum, but the scattering was higher at shorter wavelengths, which resulted in greater discrepancies for DCVI than for NDVI. Based on the results from that analysis, we decided to use the urban aerosol mixture type, due to its higher impact on NDVI/DCVI values and very high correlation with the results for the rural aerosol mixture type.

The obtained results confirmed that the most important factors which modify the measurement of vegetation indices are altitude and atmospheric optical depth. For that reason, we decided to perform a full analysis of NDVI/DCVI variability in the altitude range of $0-5 \mathrm{~km}$ and $A O D$ 0-0.25. After the simulation, differences between the real value of indices (measured at ground level) and those at every point of simulation were calculated and presented as separate figures, depicting errors of measurements made at a given altitude with a defined AOD.

Figure 10 presents the results of simulations for the NDVI index. The left panel shows the variability of the index with increasing altitude and atmospheric optical depth. Over the whole range of AODs below $1 \mathrm{~km}$ of altitude, the results were similar, presenting a barely visible decrease in values with increasing AOD. Above 2 kilometres, the AOD dependence was higher than below this altitude. The results for the analysed range showed the greater dependence of NDVI on AOD than on altitude. The right panel, dedicated to the errors of NDVI measurement, shows that in the entire area the error rate did not exceed $7.5 \%$. For altitudes below $1 \mathrm{~km}$, it was less than $1.75 \%$ and for 5 kilometres, with AODs $<0.10$, it was about $5 \%$.

The same simulations were made for DCVI, as depicted in Figure 11. Here it is notable that values of DCVI were lower and there was a sharp decrease in values with increasing altitude. DCVI was significantly more dependent on altitude than NDVI. The reason for this situation, already mentioned during the discussion of the earlier simulations, lies in the nature of Rayleigh scattering, which is significantly higher for the blue band than for the red. Rayleigh scattering wavelength dependency is driven by four factors, with a much higher scattering in short wavelengths. The simulation for DCVI clearly corresponded with that theoretical basis. On the right panel depicting errors, we notice that for the measurement from the altitude of 5 kilometres with AOD above 0.2 , the error rate reached $25 \%$ of the real value. The estimations of DCVI made from the altitudes above $4 \mathrm{~km}$ were exposed to $20-25 \%$ underestimation of the real value. Below $1 \mathrm{~km}$, the errors reached the level of $5 \%$.

The final comparison of NDVI and DCVI is presented in Figure 12, which depicts the percentage difference of errors in both methods. Firstly, we observe that the figure presents a mostly vertical gradient, which means that the main source of differences between both indices was altitude, not AOD. This means that both methods are sensitive to the increase of AOD in a similar 


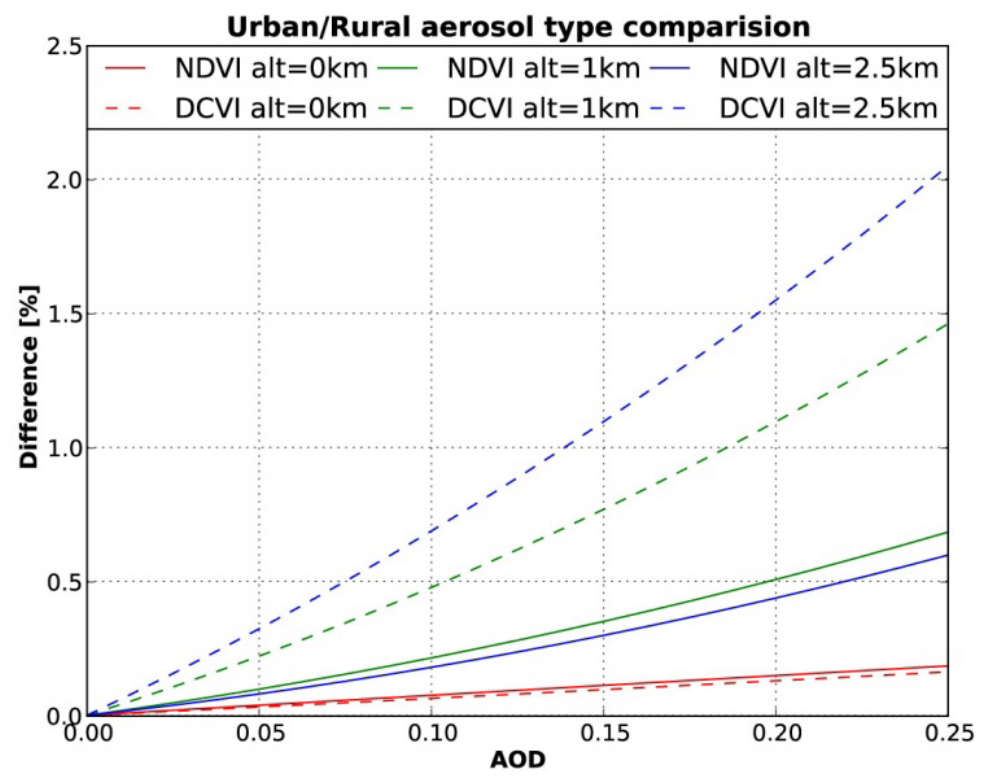

Figure 9. Differences in NDVI/DCVI results between simulations with urban and rural aerosol type

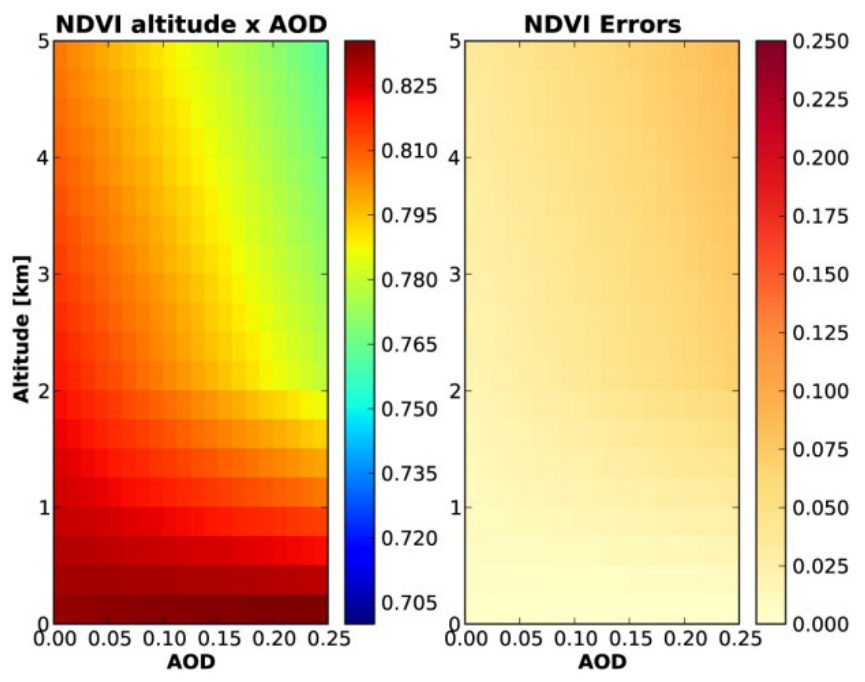

Figure 10. Variability of NDVI with altitude and AOD changes (a), differences between NDVI results at given altitude $x$ AOD and ground measurements

way, because of Mie scattering, which is the same across the whole spectra. The explanation for the differences between the indices is Rayleigh scattering, which is wavelength-dependent and increases with the optical path (altitude). Comparing the values it can be noted that, for the altitude of $5 \mathrm{~km}$, the difference reached $15 \%$, which means that by using DCVI instead of NDVI our error rate is higher by $15 \%$. Below $1 \mathrm{~km}$, both indices worked quite well and the difference between them was below $5 \%$. The comparison of absolute errors shows that DCVI error was three times higher than NDVI error.

\section{Discussion and conclusions}

The presented results of the simulations exemplify the significance of the influence the atmosphere has on remote sensing measurements in the visible and near infrared spectra used by NDVI/DCVI products. The simulations were conducted for a relatively small range of AODs and altitudes common for UAS missions. In the tested variable space, the factors with the greatest impact on the results are altitude (length of optical path) and AOD. In the analysed case, the discrepancies between the two tested standardized aerosol mixture types (Hess et al. 1998), urban and rural, reach up to $0.65 \%$ in the results for NDVI, and up to $2.04 \%$ for DCVI. This suggests that for both it is perfectly acceptable to use the same corrections. However, the aerosol composition in the atmosphere could be highly diversified: different compounds, load and vertical profiles lead to different optical properties of the atmosphere, and thus make the problem of atmospheric correction more complex (Chrysoulakis 


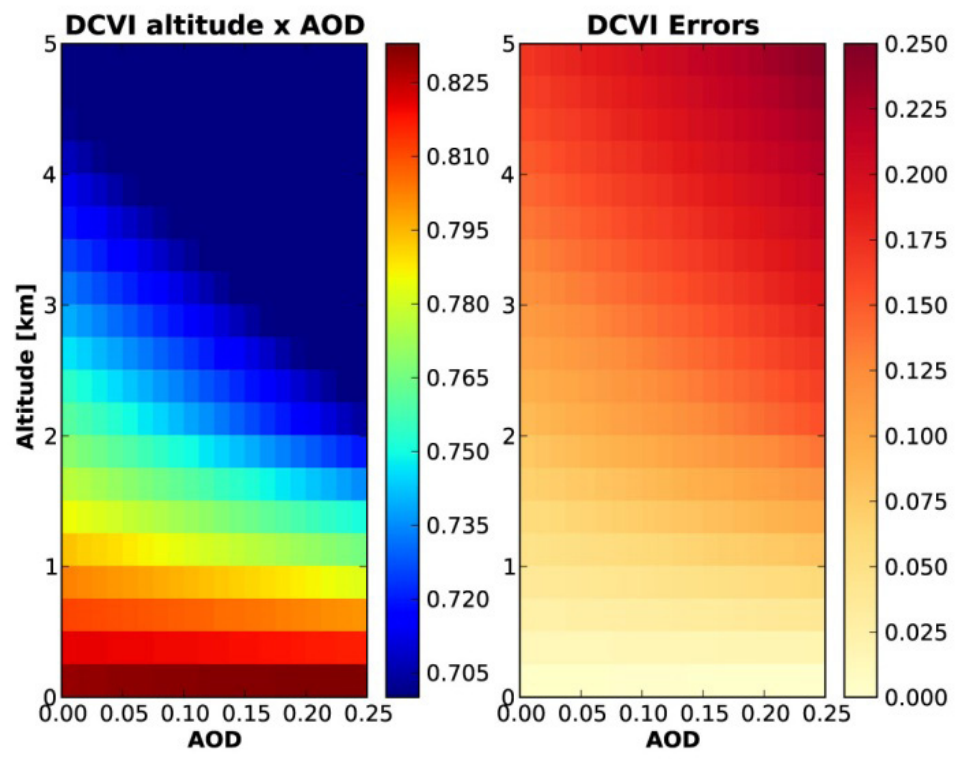

Figure 11. Variability of DCVI with altitude and AOD changes (a), differences between NDVI results at given altitude $x A O D$ and ground measurements

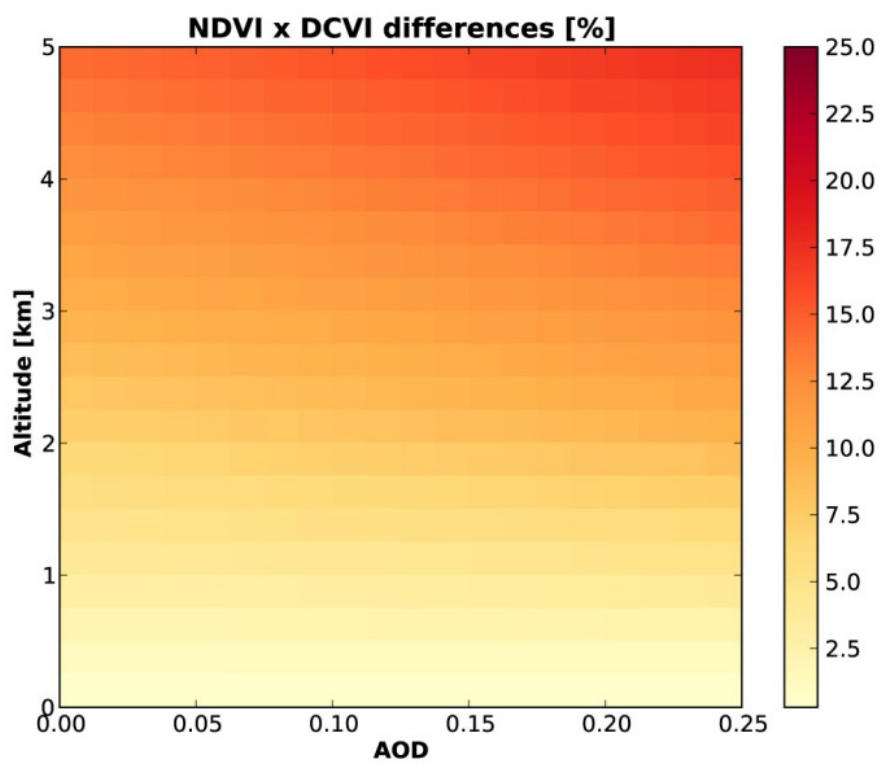

Figure 12. Variability of DCVI relative difference between ground measurement and at given altitude $x A O D$ in comparison to the same difference for NDVI

et al. 2010). Aerosols introduce absorption, Mie scattering and geometrical scattering in the visible spectrum. Determining the actual properties of aerosols in an analysed scene usually requires additional data from various satellite sensors, or from ground-based networks, such as the AErosol RObotic NETwork (AERONET), a network of sun photometers, which measures atmospheric aerosol properties every day, all year round (Holben et al. 1998). The interpretation of uncorrected data could lead to significant errors. The results show that the uncorrected NDVI product from UAS carries an error risk of about $7 \%$, while the usage of uncorrected DCVI leads to errors of about $25 \%$, which renders it useless for altitudes above $4 \mathrm{~km}$. The conclusions from the comparison of the two vegetation indices (NDVI and DCVI) show that every differential index should use bands which are as close as possible to each other, in order to minimize the wavelength-dependent effects. When choosing the appropriate band, it is important to consider atmospheric windows and regions of absorption by gases. The atmospheric contribution to the remote sensing data increases with altitude (length of optical path), view angle, aerosol load and shorter wavelengths. During the processing of data, we also have to take into account different solar zenith and azimuth angles, distance between the 
Sun and the Earth, and properties of the analysed objects. In the presented simulations, a simplified surface with grass-type albedo was assumed, whereas in real-life scenarios bidirectional reflectance distribution functions add additional complexity to the entire issue.

Despite the fact that the performed analysis revealed possible inaccuracies stemming from the uncorrected DCVI usage in the lowest part of atmosphere, it is important to consider the results from the perspective of common research into the usage of UAS. Most short flights of light UAS are below the altitude of 400 meters, and for flights at this level the optical depth of atmosphere in standard conditions only contributes up to $2.5 \%$ of error for uncorrected DCVI. This means that in most flights, when there are no events significantly increasing the load of aerosols, it is acceptable to use uncorrected data. Moreover, when a study is focused on data interpretation from one mission only, and the values of vegetation indices are compared on a single picture, even data from higher altitudes can be used. For studies based on time series and higher altitudes, it is recommended to use cameras capable of calculating NDVI instead of DCVI. DCVI can be used for most low altitude measurements (below 150 meters) For the purpose of calculating the atmospheric correction during campaigns, it would be advisable to hand-hold a sun-photometer, which can instantly and precisely measure the AOD at selected wavelengths (Markowicz et al. 2012).

The benefits of using DCVI cameras are indisputable, as far as cost effectiveness, user-friendliness and size and weight of the devices are concerned (Chiliński 2010, 2012). The presented results confirm that such devices could be successfully used in UAS for low altitude flights; however, researchers should be aware of the limitations and weaknesses of this method regarding measurements at higher altitudes.

\section{Acknowledgements}

The research is funded by the Polish Ministry of Science and Higher Education (2012-2015) under the Diamond Grant programme.

\section{References}

Anderson, G, Clough, S, Kneizys, F, Chetwynd, J \& Shettle, E 1986, 'AFGL atmospheric constituent profiles (0-120 km)', Tech. Rep. AFGL-TR-86-0110, Air Force Geophys. Lab., Hanscom Air Force Base, Bedford, Mass.

Burgheimer, J, Wilske, B, Maseyk, K, Karnieli, A, Zaady, E, Yakir, D \& Kesselmeier, J 2006, 'Relationships between Normalized Difference Vegetation Index (NDVI) and carbon fluxes of biologic soil crusts assessed by ground measurements', $J$. Arid Environ, vol. 64, pp. 651-669.

Chiliński, M 2010, Fundamentals of imaging, remote sensing method for assessing the state of vegetation and verification of its usefulness in environmental studies, Bachelor thesis on University of Warsaw.

Chiliński, M 2012, Improvement of Data Acquiring in ${ }_{L} N D V I$ Method and Comparison of it's Results with Hyperspectral Spectrometer Measurements, Master thesis on University of Warsaw.

Chrysoulakis, N, Abrams, M, Feidas, H \& Arai, K 2010, 'Comparison of atmospheric correction methods using ASTER data for the area of Crete, Greece', International Journal Of Remote Sensing, vol. 31, no. 24, pp. 6347-6385.

Fischer, T, Veste, M, Eisele, A, Bens, O, Spyra, W \& Hüttl, RF 2012, 'Small scale spatial heterogeneity of Normalized Difference Vegetation Indices (NDVIs) and hot spots of photosynthesis in biological soil crusts', Flora, vol. 207, pp. 159-167.

Hardin, PJ \& Hardin, TJ 2010, 'Small-scale remotely piloted vehicles in environmental research', Geography Compass, vol. 4, pp. 1297-1311.

Hess, M, Koepke, P \& Schult, I 1998, 'Optical Properties of Aerosols and Clouds: The Software Package OPAC', Bulletin of the American Meteorological Society, vol. 79, pp. 831-844.

The HITRAN Database 2012. Available from: <http://www.cfa harvard.edu/hitran/>. [11 October 2013].

Holben, BN, Eck, TF, Slutsker, I, Tanré, D, Buis, JP, Setzer, A, Vermote, E, Reagan, JA, Kaufman, YJ, Nakajima, T, Lavenu, F, Jankowiak, I \& Smirnov, A 1998, 'AERONET A federated instrument network and data archive for aerosol characterization', Remote Sensing of Environment, vol. 66 pp. 1-16.

Hunt, ER, Cavigelli, M, Daughtry, CST, McMurtrey, JE \& Walthall,
CL 2005, 'Evaluation of digital photography from model aircraft for remote sensing of crop biomass and nitrogen status', Precision Agriculture, vol. 6, pp. 359-37.

Ichii, K, Matsui, Y, Yamaguchi, Y \& Ogawa, K 2001, 'Comparison of global net primary production trends obtained from satellite based normalized difference vegetation index and carbon cycle model', Global Biogeochem, Cycles, vol. 15, pp. 351-364.

Kylling, A \& Stamnes, K 1992, 'Effcient yet accurate solution of the linear transport equation in the presence of internal sources: the exponential-linear-in-depth approximation', J. Com. Phys., vol. 102, pp. 265-276.

Lelong, CCD, Burger, P, Jubelin, G, Roux, B, Labbe, S \& Barett, F 2008, 'Assessment of unmanned aerial vehicles imagery for quantitative monitoring of wheat crop in small plots', Sensors, vol. 8, pp. 3557-3585.

libRatran, 2013. Available from: < http://www.libradtran.org>

Markowicz, KM, Zielinski, T, Blindheim, D, Gausa, M, Jagodnicka, AK, Kardas, A, Kumala, W, Malinowski, SP, Posyniak, M, Petelski, T \& Stacewicz, T 2012, 'Study of vertical structure of aerosol optical properties by sun photometers and ceilometer during macron campaign in 2007', Acta Geophys., vol. 60, no. 5, pp. 1308-1337.

Myneni, RB, Keeling, CD, Tucker, CJ, Asrar, G \& Nemani, RR 1997, 'Increased plant growth in the northern high latitudes from 1981 to 1991', Nature, vol. 386, pp. 698-702.

Nebiker, S, Annen, A, Scherrer, M \& Oesch, D 2008, 'A lightweight multispectral sensor for micro UAV. Opportunities for very high resolution airborne remote sensing. The International Archives of the Photogrammetry', Remote Sensing and Spatial Information Sciences, vol. XXXVII Part B1, pp. 1193-120.

Rango, A, Laliberte, AS, Herrick, JE, Winters, C, Havstad, K, Steele, C \& Browning, D 2009, 'Unmanned aerial vehicle-based remote sensing for rangeland assessment monitoring, and management', Journal of Applied Remote Sensing, vol. 3

Rouse, J, Haas, R, Well, J \& Deering, D 1973, 'Monitoring vegetation systems in the great plains with erts', In Third Earth Resources Technology Satellite-1 Symposium, vol. I, Technical Presentations Section A, pp. 309-317.

Running, SW 1990, 'Estimating terrestrial primary productivity 
by combining remote sensing and ecosystem simulation', Remote Sensing of Biosphere Functioning. Ecol. Stud., vol. 79, pp. 65-86.

Stamnes, K, S-Chee, T, Wiscombe, W \& Kolf, J 1988, 'Numerically stable algorithm for discrete-ordinate-method radiative transfer in multiple scattering and emitting layered media', Applied Optics, vol. 27, pp. 2502-2509.

Tucker, CJ, Fung, IY, Keeling, CD \& Gammon, RH 1986 'Relationship between atmospheric $\mathrm{CO}_{2}$ variations and a satellite-derived vegetation index', Nature, vol. 319, pp. 195-199.

Xiang, H \& Tian, L 2011, 'Method for automatic georeferencing aerial remote sensing (RS) images from an unmanned aerial vehicle (UAV) platform', Biosystems Engineering, vol. 108, pp. 104-111.
Wanner, W, Strahler, A, Hu, B, Lewis, P, Muller, JP, Li, X, Barker Schaaf, C \& Barnsley, M 1997, 'Global retrieval of bidirectional reflectance and albedo over land from EOS MODIS and MISR data. Theory and algorithm', J. Geophys. Res., vol. 102, pp. 17143-17161.

Zawadzka, O, Markowicz, KM, Pietruczuk, A, Zielinski, T \& Jarosławski, J 2013, 'Impact of urban pollution emitted in Warsaw on aerosol properties', Atmospheric Environment, vol. 69 , pp. 1528.

Zhang, C \& Kovacs, J 2012, 'The application of small unmanned aerial systems for precision agriculture: a review', Precision Agriculture, vol. 13, pp. 693-712. 